\title{
A FRAMEWORK FOR CLASSIFYING INTERACTIONS IN CULTURAL HERITAGE INFORMATION SYSTEMS
}

\author{
Juliane Stiller \\ Berlin School of Library and Information Science, Humboldt-Universität zu Berlin, Dorotheenstr. 26, 10117 Berlin, Germany - \\ juliane.stiller@ibi.hu-berlin.de
}

KEY WORDS: Cultural heritage information systems, interaction patterns, taxonomy, digital libraries, information access

\begin{abstract}
:
With the mass digitization of cultural heritage and the increase of people accessing the digitized memory objects, it becomes crucial to develop meaningful interaction patterns in cultural heritage information systems. This explorative study is based on an investigation of 50 websites from the cultural heritage domain. It derives a framework for classifying user interactions with digital cultural heritage. The framework has two dimensions; the first one is a classification of the interactions and the second one describes their degree of complexity. The strength of this framework is the ability to compare complexity, scope and purpose of interactions across different websites while offering a meaningful vocabulary for discussing different interaction features.
\end{abstract}

\section{INTRODUCTION}

Recently, memory institutions face the challenge to broaden access to their cultural heritage material by digitizing it and providing and displaying a digital surrogate online. They need to handle the demand for interoperable metadata and making it retrievable, in the best case across languages and cultures. Moreover, cultural institutions seek for a meaningful presentation in terms of displaying and contextualizing their digitized cultural heritage data. However, in many cases the digital representations do not reflect the context the original artifacts were embedded in which leads to a loss of meaningful information. Therefore, the task is to define purposeful interactions with cultural heritage online and give users guidance to explore new ways in dealing with these digital artifacts. In this process, it is essential to identify the potential benefits of displaying and providing cultural heritage in a digital medium. The goal is to build systems for interacting with memory artifacts that are open to evolve and can adapt to interaction and usage patterns that are not yet foreseeable.

Many recently developed cultural heritage information systems are lacking a strategy for user involvement and purpose of such an engagement. They have striking similarities to cultural heritage search engines suppressing the fact that many users might not be able to express information needs for a cultural heritage artifact in a query. In this domain, supporting serendipity and exploration of the unknown should be a more desirable interaction feature than retrieving known items.

Initiatives like Europeana ${ }^{1}$, launched to improve access to Europe's digitized cultural heritage objects and their metadata, are researching and pushing for new interaction patterns and the development of interfaces which allow for rich user experiences with their content. Nevertheless, it is very hard to design interaction patterns in this juvenile area. Some memory institutions are trailblazers in the field of digital interaction but they struggle to determine why certain features are not adopted and users refrain from interacting with them.

This explorative study aims at developing a framework, which enables comparison of different interaction features and their inherited patterns. Consequently, variations of interaction

\footnotetext{
${ }^{1}$ http://www.europeana.eu
}

features across different cultural heritage information systems can be mapped in this framework. Moreover, it reflects the state of the art of interaction features implemented in cultural heritage websites and enables the development of best practices and recommendations for advancing interactions in this domain. By reviewing a sample set of cultural heritage information systems, interaction features are grouped and categorized to reflect the spectrum of interactions available on current systems. To catch the various degrees of complexity in which the features were implemented, an additional dimension is introduced which allows grouping of interactions on an ordinal scale.

The paper is structured as follows: The next section defines the used terminology and background of the study and refers to related work. Section 3 presents the methodology of the analysis. Section 4 introduces the framework of interactions. The paper ends with a conclusion and an outlook on future work.

\section{BACKGROUND}

\subsection{Cultural heritage information systems}

Cultural heritage consists of objects created by or interpreted by humans in contrast to natural heritage (Bearman, 2002). These objects are products, which inherit a purpose and are defined by their use (Bearman, 2002). Including intangible objects such as dances or language will explicitly extend this definition.

With the rise of digitization in the last two decades, digital surrogates and their metadata, which reside in information systems online, complement cultural artifacts. Information systems need to be developed which provide novel and innovative ways to experience cultural heritage outside physical institutions, especially as memory institutions move from being gatekeepers to becoming facilitators and mediators of knowledge exchange (Freedman, 2000).

A cultural heritage information system is an information system that collects, stores, organizes, searches and displays cultural heritage objects including their metadata in a digital environment. It needs to answer questions of "who, where, why, how, when; and what was created, collected, discovered, described, published, and exhibited" (Bearmann, 2002). This 
requires the information system to provide interactions that go beyond the common search experience and accommodate contextualization and collaboration. Furthermore, a cultural heritage information system should be transparent about the scope and extend of its collections to facilitate ease of use.

\subsection{Interactions}

Interaction is a multifaceted and interdisciplinary term with slightly different meanings and intentions across domains. This study follows the definition coming from the Human-Computer Interaction (HCI) field, where an interaction includes all engagement between a human and a computer. The term interaction consists of all tasks a user can fulfill in a cultural heritage information system including searching, retrieving and browsing items. Furthermore, it includes tasks that support collaborative engagements such as editing a user profile, uploading objects and collaboratively creating collections. This is similar to the view an interaction designer takes on interactions which is also commonly referred to as interaction patterns: the desire to offer an easy-to-use and successful solution for a common web task or known problem such as logging into an account or adding a tag to a resource (Crumlish, 2009 , p. 10). The simplicity and design of such a solution plays a major role in this context where in some cases the size of a button can make the difference. Design implications are of less interest; the behavioral aspects of interaction patterns are the main focus of this study.

In recent research, interaction is put into a broader perspective. These considerations reflect on possibilities interactions offer in a digital environment forming a new medium whose potential is not yet conceivable (Murray, 2011). A paper in the field of visual analytics understands interactions as a process through which knowledge for the user is derived or altered (Pike, 2009).

To the best of the author's knowledge, there are only a limited number of publications dealing with interactions in cultural heritage information systems. Most of these studies in this field are aimed at analyzing the acceptance and usage of a certain website feature with the goal to reveal technical weaknesses and user intentions to derive best practices. Paul Marty for example analyzed user expectations when creating collections on museum sites (Marty, 2011). The focus was on determining to which extend the system features were used and for which purpose. It does not suggest how much these expectations match the actual features and some of the expectations are derived from the use of similar application in different domains.

Furthermore, Liew analyzed information retrieval features and the provided search and browsing capabilities on cultural heritage websites (Liew, 2005). Although this study is already 7 years old and has an explorative nature, most of the findings are still true for many cultural heritage websites. It states for example that many of these sites have a unified interface for all users and offer only limited implementation of multilingual search features. This observation is still valid for recently developed cultural heritage websites.

In the broader context of digital libraries, studies were conducted to better understand user interactions and derive requirements for the design. Here, interactions are influenced by design choices and follow closely the definition of interaction designers given above. One study compared different browsing and search features and their influence on the user's performance. One lesson from the experiment is that poorer design choices are leading to a drop in performance (Zhang, 2008).

Additionally, there is a range of research that describes frameworks to better understand the different dimensions of humans interacting with information. One example is the work of Belkin and Cool who developed a classification scheme for information seeking behavior (Belkin, 2002).

Marcia Bates proposed a cascade of interactions for digital libraries (Bates, 2002). She states that it is important to understand the different parts of a digital library and their interplay. Her model, theoretically dissecting a digital library into strategic parts, suggests that each component of a digital library influences the design of the part built upon. The core component of an information system constitutes of the content and the technical backbone building up on cascading layers. The last layer presents user's expectations and interactions with the system that are influenced by design and technical decisions which are made before (Bates, 2002).

In a much more simplified model derived from Bates' assumptions, it can be determined that every information system (also outside the cultural heritage domain) strives for a seamless interaction between the users and the content. The layers in between - on the one hand the system which enables access to the content in all its facets and on the other hand the interaction patterns and interface functionalities which enable the user to interact - should be as transparent and intuitive as if they were invisible to the user. Figure 1 illustrates this model.

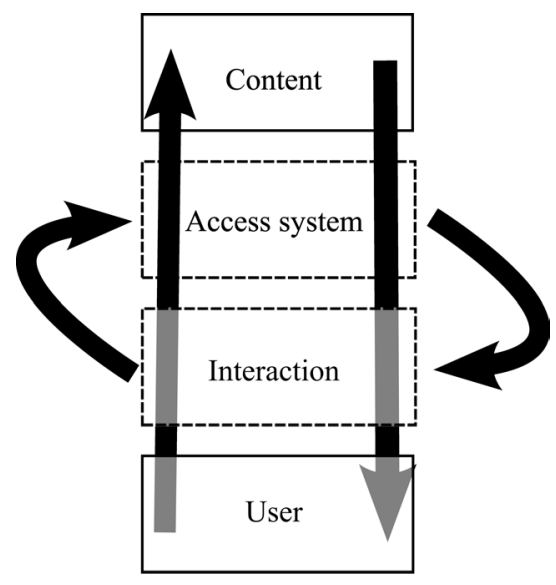

Figure 1. Model of an ideal information system

To the best of the author's knowledge a theoretical framework of interactions within digital cultural heritage has not yet been developed.

\section{METHOD}

Describing the different interaction patterns and features present on cultural heritage websites is a first step to deeper analyzing them and allowing for comparisons across sites. The cultural heritage domain is in need of a common vocabulary to be able to discuss and evaluate the different features and interactions that are implemented so far.

Due to a lack of formalized frameworks to classify interactions, a content analysis was chosen to determine prevailing engagement options. The qualitative analysis was set out to answer the following questions:

1. How can user interaction patterns and features that are implemented in cultural heritage information systems be grouped and classified?

2. How can the complexity of interactions be described acknowledging that the same feature is implemented in different ways across websites?

3. How can features and subsequently cultural heritage information systems be compared?

A sample set of 50 websites was chosen - all of them fulfilling the definition of a cultural heritage information system as given 


\section{INFORMATION SYSTEMS}

in the previous section. The goal was to find a wide variety of sites reflecting the whole spectrum of cultural heritage information systems. This purposeful sampling pursues the goal of collecting websites that either stand out in their way of presenting cultural heritage material, engaging the user, being maintained by a well-known authority or being popular for their design and interaction features. For aggregating this list and retrieving sites that meet the requirements, thematic mailing lists, conference websites as well as journals were scanned.

All interaction patterns and features found on these sample websites were listed and afterwards grouped according to their scope, extent and purpose. The result was a classification scheme for these types of websites with regard to their structure, interaction with cultural heritage objects and their collaboration among users.

The scheme was refined with every new website which was analyzed in case a new interaction feature was found. The classification was adapted accordingly. It was found that interactions are very faceted. For example, some websites do have features for social tagging of cultural heritage objects but not all of them were implemented with full sharing options. To compare different features and sites it is not enough to list that they exist but to describe their level of complexity. To address this problem, another dimension was added to the classification that describes the degree of the complexity of each class of interactions.

The following section explains the developed framework in more detail.

\section{FRAMEWORK OF DIMENSIONS OF INTERACTIONS}

This section describes the result of this study - a framework that was developed based on the interaction features and interaction patterns which were found on a sample of 50 cultural heritage websites.

\subsection{Classification of interactions}

In a first step, a classification of the different interactions found on the sample websites is developed. This is the first dimension of the framework and it provides a grouping of the interactions into features that are prevailing in cultural heritage information systems.

On a meta-level, all of the interaction patterns deployed in cultural heritage information systems can be broadly subsumed under one of the three following points:

1. Content: This comprises all features that are targeted around experiencing the content such as search, curated exhibitions or deep-zoom features.

2. User: These are features and their inherited interactions that revolve around the user management and user identity such as creating and editing user profiles.

3. Participation: These are all features allowing the user to experience cultural heritage in a personal or customized way alone or in a group of like-minded.

Table 2 shows the taxonomy of interactions with a detailed description of every class. Any user action where the user interactively uses the system can be categorized into one of the 9 classes that are tailored to the cultural heritage domain.

\begin{tabular}{|c|c|c|}
\hline Meta-Class & Class & Description \\
\hline Content & $\begin{array}{l}\text { Cultural } \\
\text { heritage }(\mathrm{CH}) \\
\text { objects }\end{array}$ & $\begin{array}{l}\text { Interaction patterns } \\
\text { supported by features related } \\
\text { to the pure content } \\
\text { aggregated in information } \\
\text { systems such as searching } \\
\text { full-text. }\end{array}$ \\
\hline Content & Curation & $\begin{array}{l}\text { Interaction patterns } \\
\text { supported by features related } \\
\text { to curated content provided } \\
\text { by the information systems' } \\
\text { authorities, e.g. browsing } \\
\text { thematic exhibitions. }\end{array}$ \\
\hline User & $\begin{array}{l}\text { User } \\
\text { representation }\end{array}$ & $\begin{array}{l}\text { Interaction patterns } \\
\text { supported by features that } \\
\text { help users to represent and } \\
\text { connect with each other e.g. } \\
\text { create user profiles. }\end{array}$ \\
\hline User & $\begin{array}{l}\text { User } \\
\text { reputation }\end{array}$ & $\begin{array}{l}\text { Interaction patterns } \\
\text { supported by features that are } \\
\text { related to user reputation } \\
\text { such as rating and starring } \\
\text { favorite objects, following of } \\
\text { other user's contributions } \\
\text { across the site. }\end{array}$ \\
\hline Participation & Storytelling & $\begin{array}{l}\text { Interaction patterns } \\
\text { supported by features } \\
\text { allowing the user to add their } \\
\text { own point of view through } \\
\text { directed and chronological } \\
\text { narration. }\end{array}$ \\
\hline Participation & $\begin{array}{l}\text { User } \\
\text { exhibitions }\end{array}$ & $\begin{array}{l}\text { Interaction patterns } \\
\text { supported by features } \\
\text { allowing users to curate } \\
\text { customized exhibitions and } \\
\text { collections. }\end{array}$ \\
\hline Participation & Annotations & $\begin{array}{l}\text { Interaction patterns } \\
\text { supported by features that } \\
\text { allow the user to add } \\
\text { additional information to } \\
\text { content such as writing } \\
\text { comments or other free text. }\end{array}$ \\
\hline Participation & Social tagging & $\begin{array}{l}\text { Interaction patterns } \\
\text { supported by features for } \\
\text { adding terms and keywords. }\end{array}$ \\
\hline Participation & User objects & $\begin{array}{l}\text { Interaction patterns } \\
\text { supported by features for } \\
\text { uploading, publishing and } \\
\text { maintaining users' content. }\end{array}$ \\
\hline
\end{tabular}

Table 2. Classification of interaction patterns and interaction features in cultural heritage websites

\subsection{The degree of complexity of interactions}

When analyzing cultural heritage digital libraries, it is important to understand that the interaction is two-fold. On the one hand, there is the information system as a whole with its different components, on the other hand, there is the user. In an ideal system the user interacts with the aggregated content in a natural and seamless way (see figure 1).

A digital surrogate of a cultural heritage item possesses a different interaction potential than the original physical object. In an online environment, there are more possibilities for interaction compared to the potential in a physical institution. In physical exhibitions, the artifacts are too scarce and valuable to 
take interaction with them beyond simply looking at the particular item. Within an online information system, three prevailing types of access determine interactions:

Search: All cultural heritage information systems make their collections accessible with textual search. Matching user queries to the metadata of an object lets the user retrieve items. Search is determined by formulating a query, scanning the result list for relevant items and finding results that might answer the information need. The challenge here is that the paradigm of the known-item search common in libraries is transferred to the cultural heritage domain. But many objects, particularly the ones coming from museums, are not sufficiently described by their metadata (e.g. acquisition numbers and dates of acquisition instead of subject headings, keywords).

Browse / Explore: Browsing features are crucial for cultural heritage information systems. They support serendipity and the discovery of unknown resources. For users, extent and scope of collections in these information systems is vague and not transparent. Therefore, innovative browsing capabilities are needed. The more the data is linked (amongst each other or to external resources) and the more its structure is exploited, the more possibilities can be offered to browse and explore the content.

Engage: The engagement level comprises all access points that are created by users adding their own content and view points, collaboratively working on content creation. Additionally, it enables exploration of the content beyond the website's scope. Examples are following enrichments links to Wikipedia or uploading user-generated content and sharing it with friends.

These three access types can be arranged in a hierarchy, where search is the access type, which needs the least amount of interactions between user and system going up to the engage level that can have an infinite number of interactions depending on the complexity of the implemented features. The more interactions are possible, the more complex the information system becomes.

To have a simple classification of interactions is not enough to compare websites and their implemented features with one another. The access type the websites offer needs to be determined and that one is driven by the complexity of interaction. To achieve this, a second dimension is introduced to complement the classification and this one determines the degree of complexity of the interactions and interactive features on a scale of ordinal values (1-5):

1. Content: On the most basal level, there is content in form of digital surrogates, born-digital objects and their metadata aggregated in an information system. With regards to complexity of interaction, this level is mainly characterized by textual search as the most basic form of accessing content; in many cases it is a simple search box. This level has the least amount of interactions.

2. Organization: On the next level, structuring the content by adhering to best practices in metadata standards enables more complex interaction patterns. Consequently, this allows simple browsing and content exploration beyond search. An implementation would be the use of rich, domain-specific data models such as CIDOC-CRM ${ }^{2}$ or the Europeana Data Model ${ }^{3}$. An example of the benefits of

\footnotetext{
${ }^{2} \mathrm{http}: / /$ www.cirdo-crm.org

${ }^{3} \mathrm{http} / / /$ pro.europeana.eu/edm-documentation
}

more structured metadata is the provision of faceted search to reduce the number of results for a query.

3. Enrichment: The next level is any form of enrichment that adds additional information to an object and links it to outside resources. Enrichment provides the user with more entry points for retrieving and exploring particular content and enables the differentiation of ambiguous terms and the identification of named entities and such. The enrichment, structure and metadata level comprise features with limited interactive complexity building upon basic textual search to more elaborate interactions such as facets.

4. Contextualization: The following more complex level is contextualization. The content gets embedded into richer and more diverse contexts. This can mean that users curate cultural heritage objects and add their meaning and interpretation or it can be the provision of storytelling functionalities. At this point, interactions become very complex and get intermixed with the need to set the right incentive for the user to participate. The technical implications for implementing contextualization are very manifold; user-generated content needs to be stored, upload functionalities provided and a quality assurance deployed. Only a handful of websites offer contextualization through user-driven data.

5. Collaboration: The most complex degree of interaction is collaboration. The focus here is on working together in groups and sharing the experience. To implement this, complex group functionalities and rights management need to be set up. Furthermore, to get users to interact with each other requires multifaceted user management and representation features.

Figure 3 shows a model of the interaction degrees and their interplay with the access points including search, browse and engage components. In general, the more complex and useroriented an information system is, the more interaction features and access points it offers.

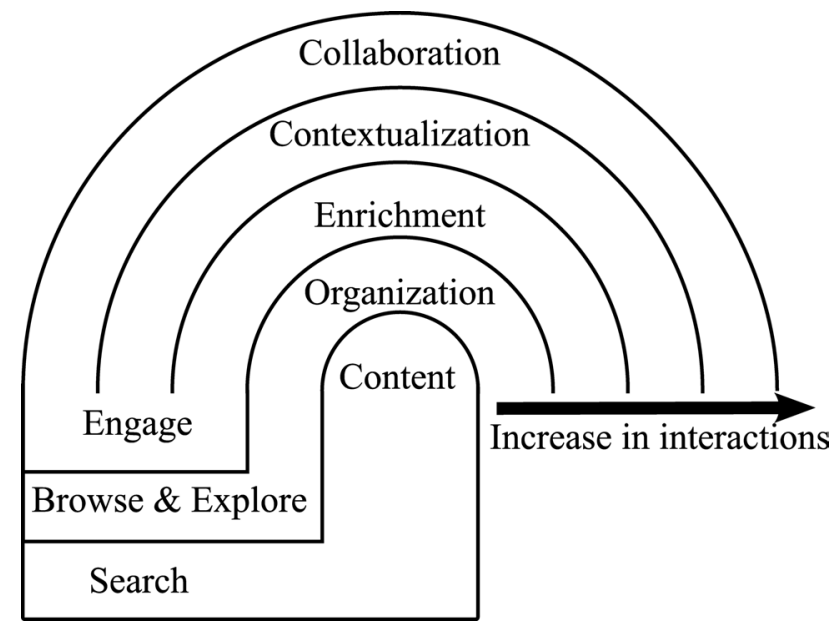

Figure 3. Degree of complexity of interactions with associated access points

This pyramid model shows that with every level the complexity of the interactions and the numbers of possible interactions increase. Every category of interaction in an information system can be analyzed in terms of its inherent interaction degree. The degree of interaction varies greatly, and different characteristics are prevailing depending on the component implemented. 


\section{INFORMATION SYSTEMS}

\subsection{Combining the classification and the degree of complexity of interactions to a framework}

Combining the classification of interactions with their degree of complexity forms a framework that enables comparison and evaluation of cultural heritage websites. It provides a means to express the complexity, degree and variability of interactions on a given site in relation to its access points. The possibility to compare interactions, features and whole information systems on an ordinal scale offers new insights and perspectives in discussing these sites.

Each class of the classification has the dimension of its complexity attached and allows to categorize each feature on a website. The focus of each cultural heritage information system becomes obvious and its implementation can be discussed. Figure 4 shows a radar model of the different classes with their complexity level. The outer rings of the radar represent more interactions than the rings closer to the center.

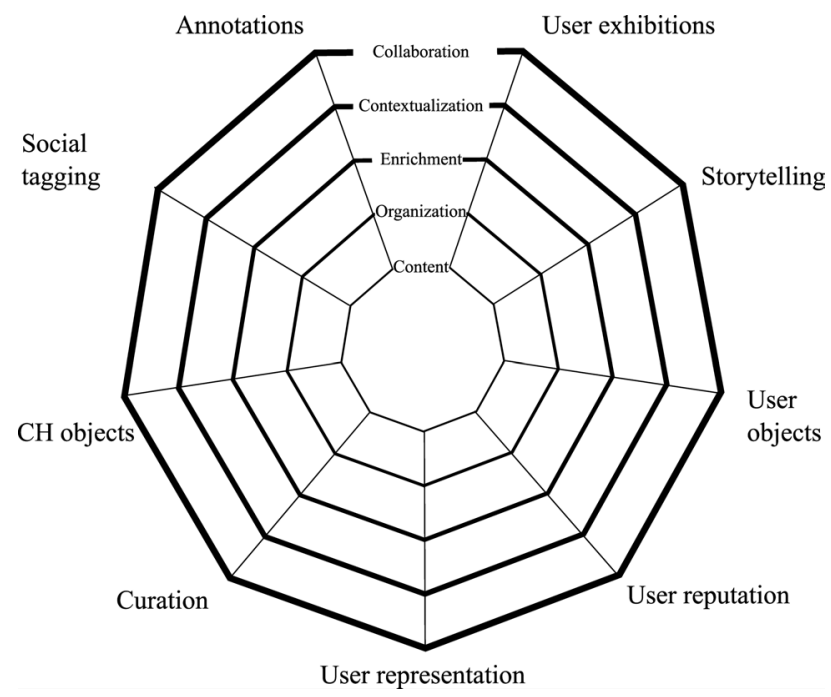

Figure 4. Model of the framework with both dimensions

For example, the interactions subsumed under the Social tagging class are integrated into many different cultural heritage websites to provide the user with a means to interact with the content but not all implementations are well engineered. For Social tagging, the different degrees of complexity would look like this:

1. Content level: This is the basis for providing a tagging feature; it comprises the tags and an appropriate storage system. In terms of interaction patterns this means: adding and deleting tags.

2. Organization level: The system enables the structuring of tags. This could mean that it stores tags with its appropriate links to the tagged resource and the tagging user. In the literature, this is referred to as the tag-resource-user relationship (Peters, 2009, p. 39). The first and second levels allow the user to search and browse the tags. Common patterns are the distinction between public and private tags and pivot browsing based on the tag-resourceuser relationship.

3. Enrichment: The system offers automatic features to ensure qualitative tags. This could mean an autocompletion feature or enrichment of the tags with controlled vocabulary.

4. Contextualization: Users are able to add complex information to the tags like descriptions, preferred terms or links. The system would also allow pivot presentation of different relations among users, tags and resources.

5. Collaboration: The most complex level comprises collaborative editing of tags and the ability to collaboratively determine preferred terms for tags excepting misspellings and outdated terms.

As shown, for each class of interaction the degree of complexity can be determined and a representational point be marked in the grid.

In a next step, a visualization of the differences between websites can be made on the basis of this framework.

One example is a comparison of Europeana's interaction features with the ones of the Google Art Project ${ }^{4}$ (figure 5). Both systems offer access to cultural heritage but their scope and intent is very different. Figure 5 shows the radar graph for these two sites. It is quite clear from this presentation that the Google Art project is much more focused on user engagement and involvement. It offers user exhibitions where a section of each picture can be added to a custom collection and be described. Furthermore, each object in the user collection can be contextualized with a Youtube video. Moreover, it is visible that it is standing out in its way of displaying its objects. It offers a deep-zoom feature that makes every stroke visible ( $\mathrm{CH}$ objects); the project adds an additional layer of experiencing cultural heritage online that goes beyond the common perception of a painting in the museum.
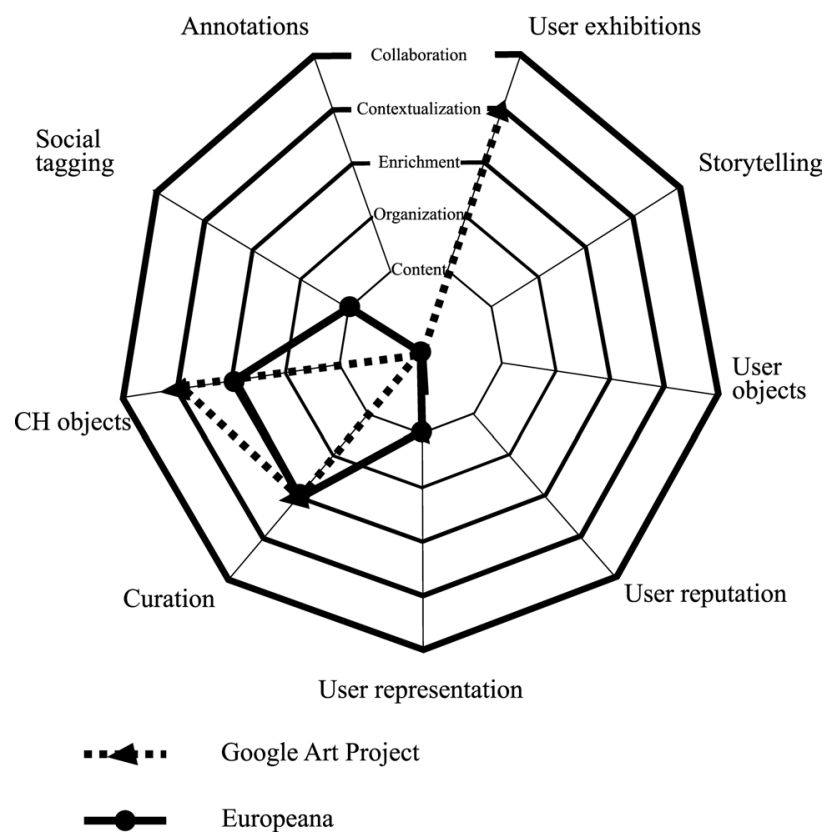

Figure 5. Comparing two websites from the sample based on the framework

Europeana, in contrast, focuses on curation of content and metadata retrieval. User engagement is only provided on a limited basis. User accounts are offered but are not used to support user participation or engagement. Europeana enables users to tag their favorite objects but it does not offer more functionalities than a private bookmarking list. In both projects, user collaboration is not implemented for any of the feature groups.

\footnotetext{
${ }^{4}$ http://www.googleartproject.com/
} 


\section{CONCLUSION AND FUTURE WORK}

In this paper, a framework for interactions on cultural heritage information systems is developed. First, a classification of different interaction groups is established and in a second step the degree of complexity of interactions determined. The framework describes the scope and purpose of each interaction and couples it with its degree of complexity. It makes it a beneficial tool for discussing different website, their desired intent and implementation. Shortcomings and implemented features can be challenged, analyzed and as a result improved. Information systems and single features across websites can be compared. The framework acts as a means to determine the focus of websites in the domain and derive best practices for matching interactions with the desired scope and purpose of cultural heritage information systems.

In future work, a quantitative analysis of the 50 websites will follow that determines which interaction patterns are occurring on these 50 websites. Additionally, best practices for implementing interactions and features will be derived from these results.

\section{References}

Bates, M. (2002). The cascade of interactions in the digital library interface. Information Processing and Management, 38(3), pp. 381-400.

Bearman, D., Trant, J., 2002. Issues in Structuring Knowledge and Services for Universal Access to Online Science and Culture, Nobel Symposium "Virtual Museums and Public Understanding of Science and Culture", May 26-29, 2002, Stockholm,

Sweden. http://www.nobelprize.org/nobel_organizations/nobelfoundatio n/symposia/interdisciplinary/ns120/lectures/bearman.pdf (accessed 16 Aug 2012)

Cool, C. and Belkin, N., 2002. A classification of interactions with information. H. Bruce (Ed.): Emerging Frameworks and Methods: CoLIS4: proceedings of the Fourth International Conference on Conceptions of Library and Information Science, Seattle, WA, USA, July 21-25, 2002, Greenwood Village, CO: Libraries Unlimited, pp. 1-15.

Crumlish, C., Malone, E., 2009. Designing social interfaces, O'Reilly, Sebastopol, Book.

Freedman, G., 2000. The Changing Nature of Museums. The Museum Journal, 43(4), pp. 295-306.

Liew, C.L. 2005. Online cultural heritage exhibitions: a survey of information retrieval features. Program, 39(1), pp. 4-24.

Murray, J.H., 2011. Inventing the Medium: Principles of Interaction Design as a Cultural Practice, MIT press, Cambridge, Book.

Marty, P.F., 2011. My lost museum: User expectations and motivations for creating personal digital collections on museum websites. Library \& Information Science Research, 33(3), pp. 211-219.

Peters, I., 2009. Folksonomies: Indexing and retrieval in Web 2.0, de Gruyter.
Pike, W.A. (et al.), 2009. The science of interaction. Information Visualization, 8(4), pp. 263-274.

Zhang, X.M. (et al.), 2008. Effects of interaction design in digital libraries on user interactions. Journal of Documentation. 64(3), pp. 438-463.

\section{Acknowledgements}

This research was partly funded by the Europeana v2.0 project (Ev2, Grant agreement number: 270902). 